\title{
Asymptotic charges from soft scalars in even dimensions
}

\author{
Miguel Campiglia and Leonardo Coito \\ Instituto de Física, Facultad de Ciencias, Montevideo 11400, Uruguay
}

(Received 16 January 2018; published 12 March 2018)

\begin{abstract}
We study asymptotic charges associated with a spin-zero analog of Weinberg's soft photon and graviton theorems in even dimensions. Simple spacetime expressions for the charges are given, but unlike gravity or electrodynamics, the symmetry interpretation for the charges remains elusive. This work is a higher dimensional extension of the four-dimensional case studied by Campiglia et al. [Phys. Rev. D 97, 046002 (2018)].
\end{abstract}

DOI: 10.1103/PhysRevD.97.066009

\section{INTRODUCTION}

Soft factorization theorems in scattering amplitudes can be understood as conservation laws of asymptotic charges; see [1] for a recent review. When the soft particle has spin 1 or 2 , the asymptotic charges are associated with gauge symmetries that are nontrivial at infinity [1]. For spin-zero soft particles one can still construct conserved asymptotic charges [2,3], but their interpretation in terms of symmetries remains incomplete. The factorization studied in [2] is a "leading" soft theorem (a spin-zero analog of Weinberg's soft theorem [4]), whereas [3] deals with factorization at subleading order in the soft scalar energy.

In the hope to improve the understanding of spin-zero asymptotic charges, here we extend the results of [2] to higher even dimensions. One of the advantages is that we can study massless $\varphi^{3}$ theory (which does not have a good null-infinity description in dimension four; see Sec. III A). This is perhaps the simplest context where to explore the question of asymptotic symmetries associated with soft scalars. As in [2] our discussion will be restricted to treelevel amplitudes. The study of loop corrections would require one to work in a theory where masslessness of the scalar is protected (as for instance the model studied in [3]).

The organization of this paper is as follows. In Sec. II we recall the charge associated with the soft-scalar theorem in [2]. In Sec. III we interpret this charge in terms of asymptotic fields at null infinity for massless $\varphi^{3}$ theory. In Sec. IV we extend the analysis to include massive fields $\psi$ coupled to the massless scalar. In Sec. V we discuss a smeared version of the charges and give a simple

Published by the American Physical Society under the terms of the Creative Commons Attribution 4.0 International license. Further distribution of this work must maintain attribution to the author(s) and the published article's title, journal citation, and DOI. Funded by SCOAP ${ }^{3}$. expression for a spacetime current. Some of the calculations are given in the appendices.

Our conventions are as follows. We take spacetime dimension to be $D=2 m+2$ with $m=2,3, \ldots$. We use mostly the plus signature spacetime metric. For simplicity in our discussion we focus on future null and time infinities; a parallel discussion applies to the past infinities. We also omit a discussion of fields at spacelike infinity. This shares many features with the study of fields at time infinity, except that differential equations become hyperbolic. In particular, it allows one to understand the conservation of asymptotic charges from a dynamical perspective [2,5].

\section{SOFT THEOREM CHARGE}

We consider a theory of a self-interacting scalar field $\varphi$ coupled to a massive scalar $\psi,{ }^{1}$

$L=-\frac{1}{2}(\partial \varphi)^{2}-\frac{1}{2}(\partial \psi)^{2}-\frac{1}{2} \mu^{2} \psi^{2}+\frac{g}{3 !} \varphi^{3}+\frac{g^{\prime}}{2 !} \psi^{2} \varphi$.

At tree level, one can obtain a "soft theorem" that relates an $(n+1)$-particle amplitude with an $n$-particle amplitude, where the $(n+1)$ th particle is a soft $\varphi$. This soft theorem can be written as matrix elements of the identity [1]

$$
Q(\hat{q}) S=S Q(\hat{q})
$$

where $S$ is the S-Matrix, $\hat{q}$ the direction of the soft $\varphi$-particle and [2]

\footnotetext{
${ }^{1}$ We could also consider $\psi$ 's with nonzero spin [2] or with zero mass (in which case the treatment will be similar to that of $\varphi^{3}$ theory). The main reason to introduce $\psi$ is to discuss how to deal with massive fields.
} 


$$
\begin{aligned}
Q(\hat{q}):= & \lim _{\omega \rightarrow 0} \frac{\omega}{2}\left(a(\omega \hat{q})+a^{\dagger}(\omega \hat{q})\right) \\
& -\frac{g}{2} \int \widetilde{d p} \frac{a^{\dagger}(\vec{p}) a(\vec{p})}{p \cdot q} \\
& -\frac{g^{\prime}}{2} \int \widetilde{d p} \frac{b^{\dagger}(\vec{p}) b(\vec{p})}{p \cdot q} .
\end{aligned}
$$

Here $a$ and $b$ are the Fock operators associated with the fields $\varphi$ and $\psi$ respectively, $\widetilde{d p}$ the standard Lorentz invariant measure and

$$
q^{\mu}=(1, \hat{q})
$$

a null, future pointing four-momentum associated with $\hat{q}$.

\section{CHARGES IN MASSLESS $\varphi^{3}$ THEORY}

In this section we set $g^{\prime}=0$ in (2.1) and restrict our attention to massless $\varphi^{3}$ theory. We remind the reader that we work in even spacetime dimension grater than four: $D=2 m+2$ with $m=2,3, \ldots$.

\section{A. Field expansion near null infinity}

We assume the standard $1 / r$ expansion off null infinity,

$$
\varphi(r, u, \hat{x})=r^{-m} \sum_{n=0}^{m-1} r^{-n} \phi_{n}(u, \hat{x})+O\left(r^{-2 m}\right) .
$$

As we shall see, the leading term $\phi_{0}$ represents the "free data" in terms of which the remaining $\phi_{n}$ 's can be expressed by recursively solving the field equations. The field equation for $\varphi$ is

$$
\square \varphi+\frac{g}{2} \varphi^{2}=0
$$

where, in retarded coordinates, the wave operator takes the form

$\square \varphi=r^{-m}\left[-2 \partial_{u} \partial_{r}\left(r^{m} \varphi\right)+\partial_{r}^{2}\left(r^{m} \varphi\right)\right]+r^{-2}\left[D^{2}-m(m-1)\right] \varphi$

with $D^{2}$ the Laplacian on the $2 m$-dimensional sphere. Plugging the expansion (3.1) in (3.2) one finds

$$
\begin{aligned}
2 n \partial_{u} \phi_{n} & =-\left[D^{2}-(m-n)(m+n-1)\right] \phi_{n-1} \\
\quad \text { for } n & =1, \ldots, m-2 .
\end{aligned}
$$

For higher $n$ one gets $O(g)$ contributions from the second term in (3.2). For $n=m-1$ this gives ${ }^{2}$

\footnotetext{
${ }^{2}$ In four space-time dimensions Eq. (3.5) implies $\phi_{0}=0$ and so the expansion is not consistent with the field equations. Relaxing the falloffs (3.1) to include logarithmic terms (such as $\log r / r$ ) does not fix things.
}

$2(m-1) \partial_{u} \phi_{m-1}=-\left[D^{2}-2(m-1)\right] \phi_{m-2}-\frac{g}{2} \phi_{0}^{2}$.

We will see that $\phi_{m-1}$ plays a role analogous to the "Bondi mass-aspect" in gravity or the "charge aspect" in Maxwell theory. In particular, its $u \rightarrow-\infty$ asymptotic value defines an angular charge density $\sigma(\hat{x})$,

$$
\sigma(\hat{x}):=\phi_{m-1}(u=-\infty, \hat{x})
$$

which, as we shall see, is conserved and encodes the same information as the charge $Q(\hat{q})$ defined in Eq. (2.3).

Before moving on, we need to specify the $u \rightarrow \pm \infty$ falloffs implicitly assumed in (3.6) and consistent with (3.5). We require

$$
\begin{aligned}
\phi_{n}(u, \hat{x}) & =O\left(|u|^{-(m-1-n+\epsilon)}\right) \\
\text { for } n & =0, \ldots, m-2, \\
\phi_{m-1}(u, \hat{x}) & =O(1)+O\left(|u|^{-\epsilon}\right)
\end{aligned}
$$

as $u \rightarrow \pm \infty$ for some $\epsilon>0$. These conditions may be thought of as a real-space version of the condition that $\omega a(\omega, \hat{q})$ has a well-defined $\omega \rightarrow 0$ limit [see Eq. (2.3) and the next subsection].

With these conditions we can now use Eqs. (3.4) and (3.5) to write $\partial_{u} \phi_{m-1}$ in terms of the free-data $\phi_{0}$. Introducing the notation

$$
\Delta_{n}:=D^{2}-(m-n)(m+n-1),
$$

one finds ${ }^{3}$

$$
\begin{aligned}
\partial_{u} \phi_{m-1}= & \frac{1}{(-2)^{m-1}(m-1) !} \prod_{n=1}^{m-1} \Delta_{n}\left[\int_{-\infty}^{u} d u\right]^{m-2} \phi_{0} \\
& -\frac{g}{4(m-1)} \phi_{0}^{2}
\end{aligned}
$$

where $\left[\int_{-\infty}^{u} d u\right]^{m-2} \phi_{0}$ denotes the $(m-2)$ th primitive of $\phi_{0}(u)$.

\section{B. Relation between $\boldsymbol{Q}(\hat{\boldsymbol{q}})$ and $\boldsymbol{\sigma}(\hat{\boldsymbol{x}})$}

In this section we use the sphere-differential operator appearing in (3.9) to relate the charge $Q(\hat{q})(2.3)$ with $\sigma(\hat{x})$ (3.6). Define the $2(m-1)$ th differential operator

$$
\mathbb{K}:=-\frac{1}{(-4 \pi)^{m}(m-1) !} \prod_{n=1}^{m-1} \Delta_{n}
$$

In Appendix $A$ we show that $\mathbb{K}$ is invertible, with the inverse given by the Green's function

\footnotetext{
${ }^{3}$ See $[6,7]$ for analogous expressions in QED.
} 


$$
\begin{aligned}
K^{-1}(\hat{q}, \hat{x}) & :=\frac{2(m-1)}{1-\hat{q} \cdot \hat{x}}, \\
\mathbb{K} K^{-1}(\hat{q}, \hat{x}) & =\delta^{(2 m)}(\hat{q}, \hat{x}) .
\end{aligned}
$$

We show below that $\mathbb{K}$ maps $Q(\hat{x})$ into $\sigma(\hat{x})$ :

$$
\sigma(\hat{x})=\mathbb{K} Q(\hat{x}),
$$

with the inverse relation

$$
Q(\hat{q})=\int_{S^{2 m}} d^{2 m} \hat{x} K^{-1}(\hat{q}, \hat{x}) \sigma(\hat{x}) .
$$

To establish these relations we compare the "soft" and "hard" parts of $Q(\hat{q})$ and $\sigma(\hat{x})$ separately. The soft and hard parts of $Q(\hat{q})$ are

$$
\begin{aligned}
Q^{\text {soft }}(\hat{q})=\lim _{\omega \rightarrow 0^{+}} \frac{\omega}{2}\left(a(\omega \hat{q})+a^{\dagger}(\omega \hat{q})\right), & \\
Q^{\text {hard }}(\hat{q})= & -\frac{g}{4(2 \pi)^{2 m}} \int_{S^{2 m}} d^{2 m} \hat{p} \\
& \times \int_{0}^{\infty} \frac{d E}{2 \pi} \frac{E^{2 m-2} a^{\dagger}(E \hat{p}) a(E \hat{p})}{-1+\hat{q} \cdot \hat{p}},
\end{aligned}
$$

where in writing $Q^{\text {hard }}(\hat{q})$ we expressed the momentum integral in spherical coordinates $\vec{p}=E \hat{p}$. For $\sigma(\hat{x})$ we start with definition (3.6) to obtain

$\sigma(\hat{x})=-\int_{-\infty}^{\infty} d u \partial_{u} \phi_{m-1}(u, \hat{x})+\phi_{m-1}(u=\infty, \hat{x})$.

As we shall see in the next section, the term $\phi_{m-1}(u=$ $\infty, \hat{x})$ accounts for massive particles but is zero in the massless theory. The term $\partial_{u} \phi_{m-1}(u, \hat{x})$ can be expressed in terms of the free-data $\phi_{0}$ by means of Eq. (3.9), yielding soft (linear in $\phi_{0}$ ) and hard (quadratic in $\phi_{0}$ ) pieces,

$$
\sigma(\hat{x})=\sigma^{\mathrm{soft}}(\hat{x})+\sigma^{\text {hard }}(\hat{x}) .
$$

To compare with $Q(\hat{q})$ we express $\sigma^{\text {soft/hard }}$ in terms of the Fourier transform of $\phi_{0}$. Defining

$$
\tilde{\phi}_{n}(E, \hat{x})=\int_{-\infty}^{\infty} \phi_{n}(u, \hat{x}) e^{i E u} d u,
$$

one finds

$$
\sigma^{\mathrm{soft}}(\hat{x})=-\lim _{E \rightarrow 0} \frac{1}{(-i E)^{m-2}(-2)^{m-1}(m-1) !} \prod_{n=1}^{m-1} \Delta_{n} \tilde{\phi}_{0}(E, \hat{x}),
$$

$\sigma^{\text {hard }}(\hat{x})=\frac{g}{2(m-1)} \int_{0}^{\infty} \frac{d E}{2 \pi} \tilde{\phi}_{0}(E, \hat{x}) \tilde{\phi}_{0}(-E, \hat{x})$.
We finally express $\tilde{\phi}_{0}(E, \hat{x})$ in terms of the Fock operator $a(E \hat{p})$. In the asymptotic future, $\varphi$ is described by the freefield expression

$$
\varphi(x) \approx \int \widetilde{d p} a(\vec{p}) e^{i p \cdot x}+\text { c.c. }
$$

where $\widetilde{d p} \equiv \frac{d^{2 m+1} \vec{p}}{(2 \pi)^{2 m+1} 2|\vec{p}|^{\mid}}$. The integral over the sphere $\hat{p}=$ $\vec{p} /|\vec{p}|$ can be evaluated via saddle point in the $r \rightarrow \infty, u=$ const limit, yielding an expansion of the type (3.1) with

$\phi_{0}(u, \hat{x})=\frac{e^{-i \pi m / 2}}{2(2 \pi)^{m}} \int_{0}^{\infty} \frac{d E}{2 \pi} E^{m-1} a(E \hat{x}) e^{-i E u}+$ c.c.

We thus obtain

$$
\tilde{\phi}_{0}(E, \hat{x})=\frac{(-i)^{m} E^{m-1}}{2(2 \pi)^{m}} a(E \hat{x}), \quad \text { for } E>0
$$

and the complex conjugated expression for $E<0$. Substituting (3.23) in (3.19) and comparing with (3.14) one finds ${ }^{4}$

$$
\sigma^{\mathrm{soft}}(\hat{x})=\mathbb{K} Q^{\mathrm{soft}}(\hat{x}) .
$$

We finally discuss the hard part of the charges. Substituting (3.23) in (3.20) yields

$$
\sigma^{\text {hard }}(\hat{x})=\frac{g}{8(m-1)(2 \pi)^{2 m}} \int_{0}^{\infty} \frac{d E}{2 \pi} E^{2 m-2} a^{\dagger}(E \hat{x}) a(E \hat{x}) .
$$

Using (3.11) it is straightforward to verify

$$
\sigma^{\operatorname{hard}}(\hat{x})=\mathbb{K} Q^{\operatorname{hard}}(\hat{x}) .
$$

This concludes the proof of Eq. (3.12). The inverse relation (3.13) follows automatically.

\section{MASSIVE FIELDS}

In this section we study contribution from massive fields. We thus take $g^{\prime} \neq 0$ in (2.1). For simplicity we set $g=0$ so that the field equation for $\varphi$ is

$$
\square \varphi=-\frac{g^{\prime}}{2} \psi^{2} .
$$

Our aim is to show that relations (3.12) and (3.13) still hold in this case. The soft part of the charges is the same as before, so we focus on the hard part of the charges. For $Q(\hat{q})$ this is given by the last term in (2.3),

\footnotetext{
${ }^{4}$ Here we wrote the $E \rightarrow 0$ limit in (3.19) with the symmetric prescription: $\lim _{E \rightarrow 0^{+}} \frac{1}{2}\left(\tilde{\phi}_{0}(E, \hat{x})+\tilde{\phi}_{0}(-E, \hat{x})\right)$.
} 


$$
Q^{\text {hard }}(\hat{q})=-\frac{g^{\prime}}{2} \int \widetilde{d p} \frac{b^{\dagger}(\vec{p}) b(\vec{p})}{p \cdot q},
$$

whereas for $\sigma(\hat{x})$ it is given by [recall Eq. (3.16)]

$$
\sigma^{\text {hard }}(\hat{x})=\phi_{m-1}(u=\infty, \hat{x}) .
$$

As in [2] we proceed by studying the field equations at timelike infinity and then compare the null and timeinfinity expansion in their common range of validity. To this end, we switch to hyperbolic coordinates

$$
x^{\mu}=\tau Y^{\mu}(y), \quad Y^{\mu}(y) Y_{\mu}(y)=-1,
$$

where $y \equiv y^{\alpha}, \alpha=1, \ldots, 2 m+1$ parametrize the unit hyperboloid $\mathcal{H}$. In these coordinates the Minkowski metric takes the form

$$
d s^{2}=-d \tau^{2}+\tau^{2} h_{\alpha \beta} d y^{\alpha} d y^{\beta}
$$

with $h_{\alpha \beta}$ the unit hyperboloid metric. We start by studying the $\tau \rightarrow \infty, Y^{\mu}=$ const behavior of the massive field $\psi$. At late times the field is described by the usual Fourier expansion

$$
\psi(x) \approx \int \widetilde{d p} b(\vec{p}) e^{i p \cdot x}+\text { c.c. },
$$

where $\widetilde{d p}=\frac{d^{2 m+1} \vec{p}}{(2 \pi)^{2 m+1} 2 \sqrt{\vec{p}^{2}+\mu^{2}}}$. In the $\tau \rightarrow \infty$ limit the momentum integral can be evaluated by a saddle-point yielding ${ }^{5}$

$$
\psi(\tau Y)=\frac{\mu^{m-1 / 2}}{2(2 \pi \tau)^{m+1 / 2}} b(\mu \vec{Y}) e^{-i \mu \tau}+\text { c.c. }+\cdots .
$$

From here we conclude that the leading $\tau \rightarrow \infty$ asymptotics of the right-hand side term in (4.1) is given by

$$
-\frac{g^{\prime}}{2} \psi^{2}=\frac{j(y)}{\tau^{2 m+1}}+\cdots
$$

with

$$
j(y)=-\frac{g^{\prime} \mu^{2 m-1}}{4(2 \pi)^{2 m+1}} b^{\dagger}(\mu \vec{Y}) b(\mu \vec{Y}) .
$$

The field equation (4.1) together with (4.8) implies a $\tau^{2 m-1}$ asymptotic falloff for $\varphi$

$$
\varphi(\tau, y)=\frac{\varphi_{\mathcal{H}}(y)}{\tau^{2 m-1}}+\cdots
$$

\footnotetext{
${ }^{5} \mathrm{Up}$ to phases which play no role in the analysis.
}

with $\varphi_{\mathcal{H}}(y)$ satisfying a Poisson-type equation on $\mathcal{H}$ :

$$
\left(\mathcal{D}^{2}+(2 m-1)\right) \varphi_{\mathcal{H}}=j .
$$

Equation (4.11) can be solved by Green's functions methods,

$$
\varphi_{\mathcal{H}}(y)=\int d^{2 m+1} y^{\prime} \sqrt{h} \mathcal{G}\left(y ; y^{\prime}\right) j\left(y^{\prime}\right)
$$

where the relevant Green's function is

$\mathcal{G}\left(y ; y^{\prime}\right)=-\frac{1}{(2 m-1) \operatorname{Vol}\left(S^{2 m}\right)}\left[-1+\left(Y \cdot Y^{\prime}\right)^{2}\right]^{-m+1 / 2}$

with

$$
\operatorname{Vol}\left(S^{2 m}\right)=2^{2 m+1} \pi^{m} \frac{m !}{(2 m) !}
$$

the volume (or area) of the unit $2 m$ sphere.

We now use (4.12) to express $\sigma^{\text {hard }}(\hat{x})(4.3)$ in terms of $j(y)$. We first choose coordinates $y^{\alpha}=(\rho, \hat{x})$ such that

$$
Y^{\mu}=\left(\sqrt{1+\rho^{2}}, \rho \hat{x}\right)
$$

with $\rho>0$ and $\hat{x}$ a unit vector parametrizing points on $S^{2 m}$. The conformal boundary of $\mathcal{H}$ is obtained by taking $\rho \rightarrow \infty$ with fixed $\hat{x}$. In Appendix B it we show that consistency of the null and time-infinity expansions for $\varphi$ implies

$$
\sigma^{\mathrm{hard}}(\hat{x})=\lim _{\rho \rightarrow \infty} \rho^{2 m-1} \varphi_{\mathcal{H}}(\rho, \hat{x}) .
$$

The right-hand side of (4.16) can be evaluated from expression (4.12) yielding

$$
\begin{aligned}
\sigma^{\operatorname{hard}}(\hat{x})= & \frac{1}{(2 m-1) \operatorname{Vol}\left(S^{2 m}\right)} \\
& \times \int d^{2 m+1} y \sqrt{h} j(y)[Y \cdot(1, \hat{x})]^{1-2 m},
\end{aligned}
$$

where $Y \cdot(1, \hat{x}) \equiv-Y^{0}+\vec{Y} \cdot \hat{x}$ is the Minkowski inner product between $Y^{\mu}$ and the null vector $(1, \hat{x})$.

We finally wish to compare (4.17) with $Q^{\text {hard }}(\hat{q})$. To this end we first express the momentum integral in (4.2) as an integral over $\mathcal{H}$ by doing the change of variable $\vec{p}=\mu \vec{Y}(y)$, resulting in

$$
Q^{\text {hard }}(\hat{q})=\int d^{2 m+1} y \sqrt{h} \frac{j(y)}{Y \cdot q} .
$$

The relation between (4.17) and (4.18) can then be established in the "inverse" form (3.13),

$$
Q^{\mathrm{hard}}(\hat{q})=\int_{S^{2 m}} d^{2 m} \hat{x} K^{-1}(\hat{q}, \hat{x}) \sigma^{\mathrm{hard}}(\hat{x})
$$


by means of a "shadow transform" identity ${ }^{6}$ [9] which in our conventions reads

$$
\begin{gathered}
\int d^{2 m} \hat{x}(1-\hat{q} \cdot \hat{x})^{-1}[Y \cdot(1, \hat{x})]^{1-2 m} \\
=\frac{(4 \pi)^{m}}{2} \frac{(m-2) !}{(2 m-2) !} \frac{1}{Y \cdot q}
\end{gathered}
$$

The converse relation $\sigma^{\text {hard }}(\hat{x})=\mathbb{K} Q^{\text {hard }}(\hat{x})$ then follows automatically.

\section{SMEARED CHARGES AND SPACETIME CURRENT}

Given a function $\lambda$ on the sphere

$$
\lambda: S^{2 m} \rightarrow \mathbb{R}
$$

we define the smeared charge by

$$
\sigma[\lambda]:=-2(m-1) \int d^{2 m} \hat{x} \lambda(\hat{x}) \sigma(\hat{x}) .
$$

[In particular, the smearing $\lambda(\hat{x})=(-1+\hat{q} \cdot \hat{x})^{-1}$ yields the charge $Q(\hat{q})$.] As in [2], we will show that the smeared charge can be written in terms of a spacetime current $j^{a}=\partial_{b} k^{a b}$,

$$
k^{a b}=\sqrt{\eta}\left(\left(\nabla^{a} \Lambda \varphi-\nabla^{a} \varphi \Lambda\right) X^{b}-(a \leftrightarrow b)\right),
$$

where $X^{a} \partial_{a}=x^{\mu} \partial_{\mu}$ is the dilatation vector field and $\Lambda$ a space-time scalar determined by $\lambda$ according to

$$
\Lambda(x)=c_{m}(-x \cdot x)^{m-1} \int d^{2 m} \hat{q} \frac{\lambda(\hat{q})}{(-q \cdot x)^{2 m-1}}
$$

with

$$
c_{m}=\frac{2(m-1)}{(2 m-1) \operatorname{Vol}\left(S^{2 m}\right)}
$$

a normalization constant. The field (5.4) satisfies the free wave equation

$$
\square \Lambda=0,
$$

with certain falloff properties we now describe.

In the $r \rightarrow \infty, u=$ const null infinity limit, one can verify (5.4) implies

\footnotetext{
${ }^{6}$ The role of the shadow transform in the context of spin 1 and 2 soft theorems was recently discussed in [8].
}

$$
\Lambda(x)=\sum_{n=1}^{m-1} r^{-n} \Lambda_{n}(u, \hat{x})+O\left(r^{-m}\right)
$$

with

$$
\Lambda_{1}(u, \hat{x})=\lambda(\hat{x}), \quad \Lambda_{n}(u, \hat{x})=O\left(u^{n-1}\right) .
$$

On the other hand, in hyperbolic coordinates adapted to timelike infinity one has

$$
\Lambda(\tau, y)=\tau^{-1} \Lambda_{\mathcal{H}}(y)
$$

with

$$
\Lambda_{\mathcal{H}}(y)=c_{m} \int d^{2 m} \hat{q} \frac{\lambda(\hat{q})}{(-q \cdot Y)^{2 m-1}}
$$

Expression (5.10) can alternatively be interpreted as the solution to the boundary-value problem

$$
\begin{aligned}
\left(\mathcal{D}^{2}+(2 m-1)\right) \Lambda_{\mathcal{H}} & =0, \\
\Lambda_{\mathcal{H}}(\rho, \hat{x}) & =\rho^{-1} \lambda(\hat{x})+O\left(\rho^{-3}\right)
\end{aligned}
$$

(see Appendix $\mathrm{C}$ for further details).

We now show that the charge (5.2) can be understood as arising from the spacetime current $j^{a}=\partial_{b} k^{a b}$. We follow the same strategy as in [10] in which one integrates the current over a $t=$ const spatial slice and evaluates the integral in the $t \rightarrow \infty$ limit. Two contributions arise in this limit, depending on whether the limit is taken along null or timelike directions. The null infinity contribution is given by

$$
\sigma_{\mathcal{I}}[\lambda]=\lim _{t \rightarrow \infty} \int d u d^{2 m} \hat{x} \partial_{u} k^{r u}
$$

where $u=t-r$ and

$k^{r u}=r^{2 m}\left[\left(\partial_{r} \Lambda \varphi-\partial_{u} \Lambda \varphi\right) u+\left(\partial_{r} \Lambda \varphi\right) r\right]-[\varphi \leftrightarrow \Lambda]$.

To evaluate (5.12) we first substitute the $1 / r$ expansions for $\varphi$ and $\Lambda$ in (5.13). This leads to a $1 / r$ expansion for $k^{r u}$ of the form

$$
k^{r u}=r^{m}\left(\sum_{n=1}^{m} r^{-n} k_{n}^{r u}\right)+O\left(r^{-1}\right)
$$

We note that $k^{r u}$ diverges in the null infinity limit. However the potentially divergent terms in (5.12) integrate to zero due to the $u \rightarrow \pm \infty$ falloffs. Indeed, from (3.7) and (5.8) one verifies 


$$
\begin{aligned}
& k_{n}^{r u}=O\left(|u|^{n-m-\epsilon}\right) \quad n=1, \ldots m-1, \\
& k_{m}^{r u}=O(1)+O\left(|u|^{-\epsilon}\right)
\end{aligned}
$$

and so the would-be divergent terms do not contribute to the charge. The finite contribution comes from

$k_{m}^{r u}(u, \hat{x})=2(m-1) \phi_{m-1}(u, \hat{x}) \lambda(\hat{x})+O\left(|u|^{-\epsilon}\right)$

and so

$$
\sigma_{\mathcal{I}}[\lambda]=2(m-1) \int d u d^{2 m} \hat{x} \lambda(\hat{x}) \partial_{u} \phi_{m-1}(u, \hat{x}) .
$$

From Eq. (3.16) we see this corresponds to the null-infinity contribution to (5.2).

We now evaluate the contribution from time infinity. To this end we switch to hyperbolic coordinates adapted to time infinity. From the $\tau \rightarrow \infty$ falloffs for $\varphi$ and $\Lambda$ one finds

$$
\lim _{\tau \rightarrow \infty} k^{\tau \alpha}=\sqrt{h}\left(\mathcal{D}^{\alpha} \varphi_{\mathcal{H}} \Lambda_{\mathcal{H}}-\mathcal{D}^{\alpha} \Lambda_{\mathcal{H}} \varphi_{\mathcal{H}}\right),
$$

leading to a time-infinity contribution to the charge:

$\sigma_{\mathcal{H}}[\lambda]=\lim _{\tau \rightarrow \infty} \int d^{2 m+1} y \partial_{\alpha} k^{\tau \alpha}=\int d^{2 m+1} y \sqrt{h} j(y) \Lambda_{\mathcal{H}}(y)$

where in the last equality we used the field equations (4.11) and (5.11).

On the other hand, according to Eq. (4.16) the "timeinfinity" contribution to (5.2) should be given by

$$
-2(m-1) \lim _{\rho \rightarrow \infty} \rho^{2 m-1} \int d^{2 m} \hat{x} \lambda(\hat{x}) \varphi_{\mathcal{H}}(\rho, \hat{x}) .
$$

That (5.20) coincides with (5.19) can be verified by writing $\varphi_{\mathcal{H}}$ in terms of $j$ according to (4.12), noting that

$$
\lim _{\rho \rightarrow \infty} \rho^{2 m-1} \mathcal{G}\left(\rho, \hat{x} ; y^{\prime}\right)=-\frac{1}{2(m-1)} \frac{c_{m}}{\left(-q \cdot Y^{\prime}\right)^{2 m-1}},
$$

and using Eq. (5.10). See Appendix C for further details.

To summarize, by evaluating the spacetime current $\partial_{b} k^{a b}$ in a $t=$ const slice with $t \rightarrow \infty$ one finds two contributions at null and timelike infinity such that their sum gives the smeared charge (5.2)

$$
\sigma[\lambda]=\sigma_{\mathcal{I}}[\lambda]+\sigma_{\mathcal{H}}[\lambda] .
$$

\section{CONCLUSIONS}

Following upon the work [2], in this paper we studied the asymptotic charges associated with a spin-zero analog of
Weinberg's soft theorem in higher even dimensions. Whereas it is relatively straightforward to recast the soft theorem as a conservation of charges $Q(\hat{q})$ in the sense of Eq. (2.2), it is less obvious how to interpret these charges in terms of asymptotic fields. Here we provided such interpretation, for the case of both massless and massive hard particles.

The interpretation of $Q(\hat{q})$ in terms of asymptotic fields allows one to obtain a spacetime expression for smeared charges. As in gravity and electrodynamics, these smeared charges can be written as integrals of total-derivative currents, $j^{a}=\partial_{b} k^{a b}$. But unlike those cases, there is so far no symmetry understanding for the tensor $k^{a b}$ : In gravity and electrodynamics $k^{a b}$ are Noether forms of local symmetries [11]; but there are no local symmetries associated with scalars. The question remains as to what is the appropriate symmetry interpretation for the soft scalar asymptotic charges.

\section{ACKNOWLEDGMENTS}

We would like to thank Alok Laddha and Sebastian Mizera for many key discussions. This work was supported by Pedeciba, Fondo Clemente Estable FCE 120141103803 , and Perimeter Institute for Theoretical Physics. Research at Perimeter Institute is supported by the Government of Canada through the Department of Innovation, Science and Economic Development and by the Province of Ontario through the Ministry of Research and Innovation.

\section{APPENDIX A: OPERATOR $\mathbb{K}$ AND EQ. (3.11)}

The operator $\mathbb{K}(3.10)$ is proportional to the composition of the $(m-1)$ differential operators:

$$
\begin{aligned}
\Delta_{n} & =D^{2}-\mu_{n} ; \\
\mu_{n} & =(m-n)(m+n-1), \\
n & =1, \ldots, m-1,
\end{aligned}
$$

where $D^{2}$ is the Laplacian on $S^{2 m}$ and $m \geq 2$. It can be easily checked that $\mu_{n}>0$ in the range (A1). Since the eigenvalues of the Laplacian $D^{2}$ are nonpositive, it then follows that zero is not an eigenvalue of $\Delta_{n}$. Thus $\Delta_{n}$ is invertible and so is $\mathbb{K}$.

To find the inverse of $\mathbb{K}$ we start by inverting $\Delta_{1}$. Its Green's function $G_{1}(\hat{q}, \hat{x})$,

$$
\Delta_{1} G_{1}(\hat{q}, \hat{x})=\delta^{(2 m)}(\hat{q}, \hat{x})
$$

is found to take the simple form ${ }^{7}$

\footnotetext{
${ }^{7}$ The reason for the simplicity of $G_{1}$ is that $\Delta_{1}$ is conformally equivalent to the flat Laplacian on $\mathbb{R}^{d}: \Delta_{1}=D^{2}-\frac{d-2}{4(d-1)} R$ with $R=d(d-1)$ the scalar curvature on $S^{d}$. In terms of $\Delta_{1}$, the operators (A1) take the simple form: $\Delta_{n}=\Delta_{1}+n(n-1)$.
} 


$$
G_{1}(\hat{q}, \hat{x})=\frac{-1}{2^{m}(m-1) \operatorname{Vol}\left(S^{2 m-1}\right)}(1-\hat{q} \cdot \hat{x})^{1-m}
$$

where

$$
\operatorname{Vol}\left(S^{2 m-1}\right)=\frac{2 \pi^{m}}{(m-1) !}
$$

is the volume of the unit $S^{2 m-1}$ sphere. We now discuss the $m=2$ and $m>2$ cases separately. For $m=2, \mathbb{K}=$ $-(4 \pi)^{-2} \Delta_{1}$ and so $K^{-1}=-(4 \pi)^{2} G_{1}=2(1-\hat{q} \cdot \hat{x})^{-1}$ which coincides with expression (3.11). For $m>2$ we use the identity [6]

$$
\Delta_{m-p}(1-\hat{q} \cdot \hat{x})^{-p}=2 p(p+1-m)(1-\hat{q} \cdot \hat{x})^{-(p+1)}
$$

from which it follows that

$$
\prod_{n=2}^{m-1} \Delta_{n}(1-\hat{q} \cdot \hat{x})^{-1}=(-1)^{m} 2^{m-2}(m-2) !^{2}(1-\hat{q} \cdot \hat{x})^{1-m} .
$$

Finally, acting with $\Delta_{1}$ on (A6) and using (A2), (A3) one arrives at the desired result

$$
\mathbb{K}(1-\hat{q} \cdot \hat{x})^{-1}=\frac{1}{2(m-1)} \delta^{(2 m)}(\hat{q}, \hat{x})
$$

\section{APPENDIX B: EQUATION (4.16)}

The asymptotic properties of $\varphi$ near null infinity described in Sec. III A imply certain asymptotic properties at time infinity which we now explore.

The idea is to consider a double $r \rightarrow \infty$ and $u \rightarrow \infty$ expansion of $\varphi$. The $r \rightarrow \infty$ expansion is the one given in (3.1) which we take to hold for all $n$ :

$$
\varphi(r, u, \hat{x})=r^{-m} \sum_{n=0}^{\infty} r^{-n} \phi_{n}(u, \hat{x}) .
$$

Each $\phi_{n}(u, \hat{x})$ has a $u \rightarrow \infty$ behavior as in (3.7),

$\phi_{n}(u, \hat{x})=\phi_{n}^{+}(\hat{x}) u^{n-(m-1)-\epsilon}+\delta_{n m-1} \sigma^{\text {hard }}(\hat{x}) \quad$ for $u \rightarrow \infty$

where we included the additional $O(1)$ term that appears when $n=m-1$ [identified as the hard charge density due to massive particles, see Eq. (4.3)]. The leading $r \rightarrow \infty, u \rightarrow \infty$ behavior of $\varphi$ is thus given by $\varphi=\sum_{n=0}^{\infty} r^{-(m+n)} u^{n-(m-1)-\epsilon} \phi_{n}^{+}(\hat{x})+r^{-(2 m-1)} \sigma^{\mathrm{hard}}(\hat{x})+\cdots$

We now wish to identify this expansion with a $\tau \rightarrow \infty$, $\rho \rightarrow \infty$ expansion at time infinity. The relation between the two sets of coordinates is

$$
r=\rho \tau, \quad u=\tau\left(\sqrt{1+\rho^{2}}-\rho\right)=\frac{\tau}{2 \rho}+O\left(\rho^{-3}\right) .
$$

Substituting in (B3) and keeping only leading terms in $\tau \rightarrow \infty, \rho \rightarrow \infty$, one finds

$$
\varphi=\frac{1}{\tau^{2 m-1} \rho^{2 m-1}} \sigma^{\operatorname{hard}}(\hat{x})+O\left(\frac{1}{\tau^{2 m-1+\epsilon}}\right)+\cdots
$$

Recalling that the $\tau \rightarrow \infty, \rho=$ const behavior of $\varphi$ is given by

$$
\varphi(\tau, \rho, \hat{x})=\frac{\varphi_{\mathcal{H}}(\rho, \hat{x})}{\tau^{2 m-1}}+O\left(\frac{1}{\tau^{2 m-1+\epsilon}}\right)
$$

we conclude from (B5) that

$$
\lim _{\rho \rightarrow \infty} \rho^{2 m-1} \varphi_{\mathcal{H}}(\rho, \hat{x})=\sigma^{\text {hard }}(\hat{x}) .
$$

\section{APPENDIX C: GREEN'S FUNCTIONS AT TIME INFINITY}

In this appendix we collect some expressions from [9,12] in a way geared to our setup. We will be slightly more general than in the rest of the paper and allow for arbitrary sphere dimension $d$ and arbitrary "conformal dimension" $h$. The case of interest in the paper is $h=1$. (More generally the value $h=1-s$ is relevant for spin-s leading soft theorem charges; see [2] for a discussion in four spacetime dimensions.)

We start by constructing a spacetime function $\Lambda(x)$ out of a function on the sphere $\lambda: S^{d} \rightarrow \mathbb{R}$ and a conformal dimension $h \in \mathbb{R}$ by $[9,12]$ :

$$
\Lambda(x):=\frac{2^{h}}{(4 \pi)^{d / 2}} \frac{\Gamma(d-h)}{\Gamma\left(\frac{d}{2}-h\right)}(-x \cdot x)^{\frac{d}{2}-h} \int d^{d} \hat{q} \frac{\lambda(\hat{q})}{(-q \cdot x)^{d-h}} .
$$

One can verify $\Lambda(x)$ satisfies the free wave equation

$$
\square \Lambda=0
$$

as well as the scaling property $t^{h} \Lambda(t x)=\Lambda(x)$. The role of the overall normalization constant becomes clear below. 
In terms of hyperbolic coordinates adapted to timelike infinity (see Sec. IV), the function takes the form

$$
\Lambda(\tau, y)=\tau^{-h} \Lambda_{\mathcal{H}}(y)
$$

where

$$
\Lambda_{\mathcal{H}}(y)=\int d^{d} \hat{q} G(y ; \hat{q}) \lambda(\hat{q})
$$

with

$$
G(y ; \hat{q})=\frac{2^{h}}{(4 \pi)^{d / 2}} \frac{\Gamma(d-h)}{\Gamma\left(\frac{d}{2}-h\right)}(-q \cdot Y)^{h-d} .
$$

From Eqs. (C2) and (C3) it follows that $\Lambda_{\mathcal{H}}$ satisfies

$$
\left(\mathcal{D}^{2}+h(d-h)\right) \Lambda_{\mathcal{H}}=0
$$

on $\mathcal{H}$. On the other hand, Eq. (C4) implies $[9,12]$

$$
\lim _{\rho \rightarrow \infty} \rho^{h} \Lambda_{\mathcal{H}}(\rho, \hat{x})=\lambda(\hat{q}) .
$$

In other words, $G(y ; \hat{q})$ is the Green's function for the boundary value problem given by Eqs. (C6) and (C7).

At time infinity there are also fields $\varphi_{\mathcal{H}}$ that capture the contribution to charges due to massive particles $[2,5,10,13]$.
These fields are in a sense "dual" to $\Lambda_{\mathcal{H}}$ and satisfy Poisson-type equations

$$
\begin{aligned}
\left(\mathcal{D}^{2}+h(d-h)\right) \varphi_{\mathcal{H}} & =j ; \\
\varphi_{\mathcal{H}}(\rho, \hat{x}) & \stackrel{\rho \rightarrow \infty}{=} O\left(1 / \rho^{d-h}\right),
\end{aligned}
$$

where $j$ is a source term that is quadratic in the massive particle Fock operators.

If $\mathcal{G}\left(y ; y^{\prime}\right)$ is the Green's function for the elliptic problem (C8) then its $\rho \rightarrow \infty$ asymptotic value is related to the Green's function (C5) by [14]

$$
\lim _{\rho \rightarrow \infty} \rho^{d-h} \mathcal{G}\left(\rho, \hat{x} ; y^{\prime}\right)=-\frac{1}{(d-2 h)} G\left(y^{\prime} ; \hat{x}\right),
$$

a well-known fact in the AdS/CFT literature.

For the case of interest in the present paper we set $h=1$ and $d=2 \mathrm{~m}$. In this case $\mathcal{G}$ is given by Eq. (4.13), $G$ can be read-off from Eq. (5.10) [or alternatively from Eq. (C5)], and relation (C9) becomes

$$
\lim _{\rho \rightarrow \infty} \rho^{2 m-1} \mathcal{G}\left(\rho, \hat{x} ; y^{\prime}\right)=-\frac{1}{2(m-1)} G\left(y^{\prime} ; \hat{x}\right)
$$

which corresponds to Eq. (5.21) in the body of the paper.
[1] A. Strominger, Lectures on the infrared structure of gravity and gauge theory, arXiv:1703.05448.

[2] M. Campiglia, L. Coito, and S. Mizera, Can scalars have asymptotic symmetries?, Phys. Rev. D 97, 046002 (2018).

[3] Y. Hamada and S. Sugishita, Soft pion theorem, asymptotic symmetry and new memory effect, J. High Energy Phys. 11 (2017) 203.

[4] S. Weinberg, Infrared photons and gravitons, Phys. Rev. 140, B516 (1965).

[5] M. Campiglia and R. Eyheralde, Asymptotic $U(1)$ charges at spatial infinity, J. High Energy Phys. 11 (2017) 168.

[6] M. Mirbabayi and M. Simonovi, Weinberg soft theorems from Weinberg adiabatic modes, arXiv:1602.05196.

[7] D. Kapec, V. Lysov, and A. Strominger, Asymptotic symmetries of massless QED in even dimensions, arXiv: 1412.2763.
[8] D. Kapec and P. Mitra, A $d$-dimensional stress tensor for Mink $_{d+2}$ gravity, arXiv:1711.04371.

[9] S. Pasterski and S. H. Shao, Conformal basis for flat space amplitudes, Phys. Rev. D 96, 065022 (2017).

[10] M. Campiglia and A. Laddha, Asymptotic symmetries of QED and Weinberg's soft photon theorem, J. High Energy Phys. 07 (2015) 115.

[11] S. G. Avery and B. U. W. Schwab, Noethers second theorem and Ward identities for gauge symmetries, J. High Energy Phys. 02 (2016) 031.

[12] J. de Boer and S. N. Solodukhin, A Holographic reduction of Minkowski space-time, Nucl. Phys. B665, 545 (2003).

[13] M. Campiglia and A. Laddha, Asymptotic symmetries of gravity and soft theorems for massive particles, J. High Energy Phys. 12 (2015) 094.

[14] J. Penedones, TASI lectures on AdS/CFT, arXiv:1608.04948. 